\title{
Subgame perfect implementation: A new result
}

\author{
Haoyang $\mathrm{Wu}^{*}$
}

\begin{abstract}
This paper concerns what will happen if quantum mechanics is concerned in subgame perfect implementation. The main result is: When additional conditions are satisfied, the traditional characterization on subgame perfect implementation shall be amended by virtue of a quantum stage mechanism. Furthermore, by using an algorithmic stage mechanism, this amendment holds in the macro world too.
\end{abstract}

Key words: Mechanism design; Subgame perfect implementation; Quantum game theory.

\section{Introduction}

The theory of mechanism design plays an important role in economics. Maskin [1] provides an almost complete characterization of social choice rules that are Nash implementable when the number of agents is at least three. Moore and Repullo [2], Abreu and Sen [3] gave the framework of subgame perfect implementation. Vartiainen [4] proposed a full characterization on subgame perfect implementation.

Recently, Wu [5] propose that when an additional condition is satisfied, the sufficient conditions for Nash implementation shall be amended by virtue of a quantum mechanism. Although current experimental technologies restrict the quantum mechanism to be commercially available [6], Wu [7] propose an algorithmic mechanism by which agents can benefit from the quantum mechanism immediately when the number of agents is not very large (e.g., less than 20). Furthermore, the traditional results on two-agent Nash implementation and Bayesian implementation are revised [8,9].

* Wan-Dou-Miao Research Lab, Shanghai, China.

Email addresses: hywch@mail.xjtu.edu.cn, Tel: 86-18621753457 (Haoyang $\mathrm{Wu})$. 
Following the aforementioned works, this paper investigates what will happen if the quantum mechanics is generalized to subgame perfect implementation. The rest of this paper is organized as follows: Section 2 recalls preliminaries of subgame perfect implementation given by Abreu and Sen [3]. In Section 3 , a novel condition multi- $\alpha$ is defined. In addition, we give an example of a Pareto-inefficient social choice correspondence (SCC) that can be implemented in subgame perfect equilibrium. Section 4 and 5 are the main parts of this paper, in which we will propose quantum and algorithmic stage mechanisms respectively. Section 6 draws the conclusions.

\section{Preliminaries}

Let $J=\{1, \cdots, N\}$ be the set of individuals. Let $A$ denote the set of outcomes. The set of preference profiles is denoted by $\Theta$. For a profile $\theta \in \Theta$, agent $j \in J$ has preference ordering $R^{j}(\theta)$ on the set $A$. Let $P^{j}(\theta)$ and $I^{j}(\theta)$ be the strict preference relation and the indifference relation associated with $R^{j}(\theta)$, respectively. For each profile $\theta \in \Theta$, an SCC $f$ picks a non-empty subset $f(\theta)$ of the set $A$. An extensive form mechanism is an array $\Gamma=(K, P, U, C, h) . K$ is the game tree with origin $n_{0}$. The set of non-terminal nodes of $K$ is denoted by $M$. The player, information and choice partitions are denoted $P, U$ and $C$ respectively. The function $h$ is the payoff function and associates with every path in the tree an element of the set $A$.

For any profile $\theta \in \Theta$, the pair $(\Gamma, \theta)$ constitutes an extensive form game. A pure strategy for player $j$ is a function which specifies a choice at every information set of player $j$. The set of pure strategies for player $j$ is denoted $S^{j}$. A pure strategy profile is an $N$-tuple of pure strategies, one for each player. The set of pure strategy profiles is denoted $S=S^{1} \times \cdots \times S^{N}$. Let $M^{0}$ denote the set of all nodes which are roots of subgames in $\Gamma$. A subgame is identified by its root $m \in M^{0}$. For all $j \in J$ and $s \in S$, the set $\Sigma(j, s)$ is defined by $\Sigma(j, s)=\left\{s^{\prime} \in S \mid s_{i}^{\prime}=s_{i}, i \neq j\right\}$. Thus, $\Sigma(j, s)$ is the set of strategy profiles in which players other than $j$ play according to $s$.

For all nodes $n$ and $s \in S$, let $a(n, s)$ denote the outcome obtained under $s$, conditional on starting at node $n$. For all nodes $n, s \in S$ and $j \in J$, let $A(j, n, s)=\left\{a\left(n, s^{\prime}\right) \mid s^{\prime} \in \Sigma(j, s)\right\}$. The strategy profile $s \in S$ is a subgame perfect equilibrium $(S P E)$ of the game $(\Gamma, \theta)$ if for every subgame $m \in M^{0}$ and every agent $j \in J, a(m, s)$ is $\theta$-maximal for player $j$ in $A(j, m, s)$. Let $\operatorname{SPE}(\Gamma, \theta)$ denote the set of subgame perfect equilibrium outcomes of $(\Gamma, \theta)$. Let $\overline{S P E}(\Gamma, \theta)$ denote the set of subgame perfect equilibrium strategy profiles of $(\Gamma, \theta)$. The SCC $f$ is subgame perfect implementable if there exists an extensive form mechanism $\Gamma$ such that $\operatorname{SPE}(\Gamma, \theta)=f(\theta)$ for all $\theta \in \Theta$. 
An SCC $f$ satisfies no-veto power (NVP) with respect to $B \subseteq A$ if $\forall \theta \in \Theta$, $\forall a \in B,\left[a R^{j}(\theta) b\right.$ for all $b \in B$ and $j \in K$, where $K \subseteq J$ and $\left.|K| \geq N-1\right] \Rightarrow$ $a \in f(\theta)$. An SCC $f$ satisfies monotonicity if for every pair of profiles $\theta, \phi \in \Theta$, and for every $a \in f(\theta)$, whenever $a R^{j}(\theta) b$ implies $a R^{j}(\phi) b(\forall j \in J)$, one has that $a \in f(\phi)$ [10]. An SCC $f$ satisfies Condition $\alpha$ with respect to the set $B \subseteq A$ if range $f \subseteq B$, and if for all profiles $\theta, \phi \in \Theta$ and outcomes $a \in f(\theta)-f(\phi)$, there exist a sequence of agents $j(\theta, \phi ; a) \equiv(j(0), \cdots, j(l))$ and a sequence of outcomes $a_{0}, a_{1}, \cdots, a_{l}, a_{l+1}$ in $B$ (where $a_{0}=a$ ), such that:

(i) $a_{k} R^{j(k)}(\theta) a_{k+1}, k=0, \cdots, l$;

(ii) $a_{l+1} P^{j(l)}(\phi) a_{l}$;

(iii) $a_{k}$ is not $\phi$-maximal for $j(k)$ in $B, k=0, \cdots, l$;

(iv) if $a_{l+1}$ is $\phi$-maximal in $B$ for all agents except $j(l)$, then either $l=0$ or $j(l-1) \neq j(l)$.

Theorem 1: If $f$ is subgame perfect implementable, then $f$ satisfies Condition $\alpha$ with respect to some $B \subseteq A$. Let $N \geq 3$. If $f$ satisfies Condition $\alpha$ and NVP with respect to some $B \subseteq A$, then $f$ is subgame perfect implementable.

To facilitate the following discussion, here we cite the stage mechanism used to implement $f$ as follows (P298, [3]).

First Stage (Stage 0): Each agent $j \in J$ announces a triplet $\left(\theta^{j}, a^{j}, n^{j}\right)$, where $\theta^{j} \in \Theta, a^{j} \in A$, and $n^{j}$ is a non-negative integer:

Rule (1): If $N-1$ agents announce the same pair $\theta, a \in f(\theta)$, the outcome is $a$, unless the remaining agent $i$ announces $\phi$, where $a \in f(\theta)-f(\phi)$ and $i=j(0)$ in the sequence $j(\theta, \phi ; a)$. In the latter event, go to Stage 1 .

Rule (2): In all other cases, the agent who announces the highest integer (break ties in favor of the agent with the lowest index) can select any outcome in $B$. Subsequent Stages (Stage $k, k=1, \cdots, l$ ): Each agent $j \in J$ can either raise a "flag" or announce a non-negative integer:

Rule (3): If at least $N-1$ agents raise flags, the agent $j(k-1)$ (in the sequence $j(\theta, \phi ; a))$ can select any outcome in $B$.

Rule (4): If at least $N-1$ agents announce zero, the outcome is $a_{k}$, unless $j(k)$ does not announce zero, in which case go to the next stage, or, if $k=l$, implement $a_{l+1}$.

Rule (5): In all other cases, the agent who announces the highest integer can select any outcome in $B$.

\section{Condition multi- $\alpha$}

Definition 1: An SCC $f$ satisfies Condition multi- $\alpha$ with respect to the set $B \subseteq A$ if range $f \subseteq B$, and for two profiles $\theta, \phi \in \Theta, a \in f(\theta)-f(\phi)$, there exist $2 \leq \Delta \leq N$ sequences of agents $j^{\delta}(\theta, \phi ; a) \equiv\left(j^{\delta}(0), \cdots, j^{\delta}\left(l^{\delta}\right)\right)$ (where $1 \leq \delta \leq \Delta$, and no agent belongs to two or more sequences), and $\Delta$ sequences 
of outcomes $a_{0}^{\delta}, a_{1}^{\delta}, \cdots, a_{l^{\delta}}^{\delta}, a_{l^{\delta}+1}^{\delta}$ (where $1 \leq \delta \leq \Delta, a_{0}^{\delta}=a$ ) in $B$, such that for every $1 \leq \delta \leq \Delta$,

(i) $a_{k}^{\delta} R^{j^{\delta}(k)}(\theta) a_{k+1}^{\delta}, k=0, \cdots, l^{\delta}$.

(ii) $a_{l^{\delta}+1}^{\delta} P^{j^{\delta}\left(l^{\delta}\right)}(\phi) a_{l^{\delta}}^{\delta}$.

(iii) $a_{k}^{\delta}$ is not $\phi$-maximal for $j^{\delta}(k)$ in $B, k=0, \cdots, l^{\delta}$.

(iv) If $a_{l^{\delta}+1}^{\delta}$ is $\phi$-maximal in $B$ for all agents except $j^{\delta}\left(l^{\delta}\right)$, then either $l^{\delta}=0$, or $j^{\delta}\left(l^{\delta}-1\right) \neq j^{\delta}\left(l^{\delta}\right)$.

Table 1: A Pareto-inefficient SCC $f$ that satisfies NVP and Condition $\alpha$.

Profile $\theta_{1} \quad$ Profile $\theta_{2}$

\begin{tabular}{cccccccc} 
Alice & Apple & Lily & Cindy & Alice & Apple & Lily & Cindy \\
\hline$u_{4}$ & $u_{1}$ & $u_{4}$ & $u_{1}$ & $u_{4}$ & $u_{1}$ & $u_{3}$ & $u_{1}$ \\
$u_{2}$ & $u_{3}$ & $u_{3}$ & $u_{3}$ & $u_{1}$ & $u_{2}$ & $u_{1}$ & $u_{4}$ \\
$u_{1}$ & $u_{4}$ & $u_{1}$ & $u_{2}$ & $u_{2}$ & $u_{4}$ & $u_{2}$ & $u_{3}$ \\
$u_{3}$ & $u_{2}$ & $u_{2}$ & $u_{4}$ & $u_{3}$ & $u_{3}$ & $u_{4}$ & $u_{2}$ \\
\hline \multicolumn{5}{c}{$f\left(\theta_{1}\right)=\left\{u_{1}\right\}$} & & \multicolumn{5}{c}{$f\left(\theta_{2}\right)=\left\{u_{2}\right\}$} \\
\hline
\end{tabular}

Example 1: Consider the SCC $f$ specified in Table 1. $J=\{$ Alice, Apple, Lily, Cindy $\}, A=\left\{u_{1}, u_{2}, u_{3}, u_{4}\right\}, \Theta=\left\{\theta_{1}, \theta_{2}\right\}$. Let $B=A$. $f$ is Pareto-inefficient from the viewpoints of agents because in profile $\theta_{2}$, each agent $j \in J$ prefers a Pareto-efficient outcome $u_{1}$ to $u_{2} \in f\left(\theta_{2}\right)$. Obviously, $f$ satisfies NVP. Consider $u_{1} \in f\left(\theta_{1}\right)$, for every $j \in J$, whenever $u_{1} R^{j}\left(\theta_{1}\right) b$ implies $u_{1} R^{j}\left(\theta_{2}\right) b(b \in A)$, but $u_{1} \notin f\left(\theta_{2}\right)$. Therefore, $f$ does not satisfy monotonicity and is not Nash implementable [1].

Now let us check whether $f$ satisfies Condition $\alpha$ and multi- $\alpha$. Consider two profiles $\theta_{1}$ and $\theta_{2}$, and outcome $u_{1} \in f\left(\theta_{1}\right)-f\left(\theta_{2}\right)$, then $\Delta=2$.

1) For the case of $\delta=1, l^{1}=1, j^{1}(0)=$ Alice, $j^{1}(1)=$ Apple; $a_{0}^{1}=u_{1}$, $a_{1}^{1}=u_{3}, a_{2}^{1}=u_{2}$.

(i): $a_{0}^{1} R^{j^{1}(0)}\left(\theta_{1}\right) a_{1}^{1}, a_{1}^{1} R^{j^{1}(1)}\left(\theta_{1}\right) a_{2}^{1}$, since $u_{1} R^{\text {Alice }}\left(\theta_{1}\right) u_{3}, u_{3} R^{\text {Apple }}\left(\theta_{1}\right) u_{2}$;

(ii): $a_{2}^{1} P^{j^{1}(1)}\left(\theta_{2}\right) a_{1}^{1}$, since $u_{2} P^{\text {Apple }}\left(\theta_{2}\right) u_{3}$;

(iii): $a_{0}^{1}$ and $a_{1}^{1}$ are not $\theta_{2}$-maximal for $j^{1}(0)$ and $j^{1}(1)$ in $B$ respectively, since $u_{1}$ and $u_{3}$ are not $\theta_{2}$-maximal for Alice and Apple in $B$ respectively;

(iv): $a_{2}^{1}$ (i.e., $u_{2}$ ) is not $\theta_{2}$-maximal in $B$ for all agents except $j^{1}(1)$ (i.e., Apple). Hence, (i)-(iv) are satisfied.

2) For the case of $\delta=2, l^{2}=1, j^{2}(0)=$ Lily, $j^{2}(1)=$ Cindy; $a_{0}^{2}=u_{1}, a_{1}^{2}=u_{2}$, $a_{2}^{2}=u_{4}$.

(i): $a_{0}^{2} R^{j^{2}(0)}\left(\theta_{1}\right) a_{1}^{2}, a_{1}^{2} R^{j^{2}(1)}\left(\theta_{1}\right) a_{2}^{2}$, since $u_{1} R^{\text {Lily }}\left(\theta_{1}\right) u_{2}$, and $u_{2} R^{\text {Cindy }}\left(\theta_{1}\right) u_{4}$.

(ii): $a_{2}^{2} P^{j^{2}(1)}\left(\theta_{2}\right) a_{1}^{2}$, since $u_{4} P^{\operatorname{Cindy}}\left(\theta_{2}\right) u_{2}$;

(iii): $a_{0}^{2}$ and $a_{1}^{2}$ are not $\theta_{2}$-maximal for $j^{2}(0)$ and $j^{2}(1)$ in $B$ respectively, since $u_{1}$ and $u_{2}$ are not $\theta_{2}$-maximal for Lily and Cindy in $B$ respectively. 
(iv): $a_{2}^{2}$ (i.e., $\left.u_{4}\right)$ is not $\theta_{2}$-maximal in $B$ for all agents except $j^{2}(1)$ (i.e., Cindy). Hence, (i)-(iv) are satisfied. Therefore, $f$ satisfies Condition $\alpha$ and multi- $\alpha$.

Given profile $\theta_{2}$, since $u_{1}$ is Pareto-efficient for all agents, it looks reasonable that in Stage 0 each agent $j \in J$ announces $\left(\theta_{1}, u_{1}, *\right)$ in order to obtain $u_{1}$ by rule (1) (where $*$ stands for any legal value). But according to Line 19 and the last line of Page 294 [3], agent $j^{1}(0)=$ Alice has incentives to unilaterally deviate from $\left(\theta_{1}, u_{1}, *\right)$ to $\left(\theta_{2}, *, *\right)$ in order to obtain $u_{4}$, which is $\theta_{2}$-maximal for Alice in $B$ (See Page 294, the third line to the last, [3]). Similarly, agent $j^{2}(0)=$ Lily also has incentives to unilaterally deviate from $\left(\theta_{1}, u_{1}, *\right)$ to $\left(\theta_{2}, *, *\right)$ in order to obtain $u_{3}$.

Note that either Alice or Lily can certainly obtain her expected outcome only if one of them deviates. Since all agents are rational and self-interested, nobody is willing to give up and let the others benefit. Therefore, both Alice and Lily will deviate from $\left(\theta_{1}, u_{1}, *\right)$ to $\left(\theta_{2}, *, *\right)$. Hence, the Pareto-efficient outcome $u_{1}$ cannot be implemented in subgame perfect equilibrium. It should be noted that in the end, rule (2) will be triggered and the final outcome is uncertain among $B$.

\section{A quantum stage mechanism}

As we have seen in Example 1, although the SCC $f$ is Pareto-inefficient from the viewpoints of agents, $f$ is subgame perfect implementable according to the stage mechanism [3]. Following Ref. [5], we will propose a $(l+1)$-stage quantum mechanism that let the Pareto-efficient outcome $u_{1}$ be implemented in subgame perfect equilibrium in profile $\theta_{2}$. The difference between the quantum and traditional stage mechanisms is the way by which each agent $j \in J$ submits $\left(\theta^{j}, a^{j}, n^{j}\right)$ to the designer in Stage 0 .

\subsection{Assumptions}

According to Eq (4) in Ref. [11], two-parameter quantum strategies are drawn from the set:

$$
\hat{\omega}(\xi, \eta) \equiv\left[\begin{array}{cc}
e^{i \eta} \cos (\xi / 2) & i \sin (\xi / 2) \\
i \sin (\xi / 2) & e^{-i \eta} \cos (\xi / 2)
\end{array}\right],
$$

$\hat{\Omega} \equiv\{\hat{\omega}(\xi, \eta): \xi \in[0, \pi], \eta \in[0, \pi / 2]\}, \hat{J} \equiv \cos (\gamma / 2) \hat{I}^{\otimes \Delta}+i \sin (\gamma / 2) \hat{\sigma}_{x}^{\otimes \Delta}$, where $\gamma$ is an entanglement measure, and $\Delta$ is specified in Condition multi- $\alpha$. $\hat{I} \equiv \hat{\omega}(0,0), \hat{D}_{\Delta} \equiv \hat{\omega}(\pi, \pi / \Delta), \hat{C}_{\Delta} \equiv \hat{\omega}(0, \pi / \Delta)$. Denote by $\mathbb{Z}_{+}$the set of non-negative integer. 


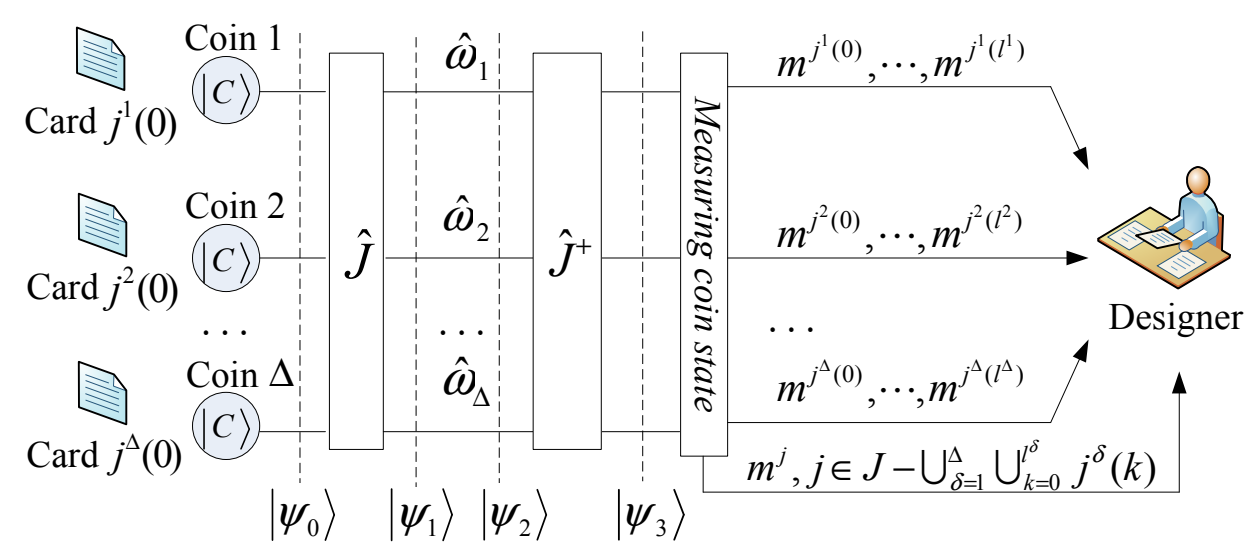

Fig. 1. The setup of a quantum stage mechanism. Each agent $j^{\delta}(0), \quad \delta=1, \cdots, \Delta$ has a quantum coin and a card. Each agent independently performs a local unitary operation on his/her own quantum coin.

Without loss of generality, we assume that:

1) Each agent $j^{\delta}(0)(1 \leq \delta \leq \Delta)$ has a quantum coin $\delta$ (qubit) and a classical card $j^{\delta}(0)$. The basis vectors $|C\rangle=(1,0)^{T},|D\rangle=(0,1)^{T}$ of a quantum coin denote head up and tail up respectively.

2) Each agent $j^{\delta}(0)(1 \leq \delta \leq \Delta)$ independently performs a local unitary operation on his/her own quantum coin. The set of agent $j^{\delta}(0)$ 's operation is $\hat{\Omega}_{\delta}=\hat{\Omega}$. A strategic operation chosen by agent $j^{\delta}(0)$ is denoted as $\hat{\omega}_{\delta} \in \hat{\Omega}_{\delta}$. If $\hat{\omega}_{\delta}=\hat{I}$, then $\hat{\omega}_{\delta}(|C\rangle)=|C\rangle, \hat{\omega}_{\delta}(|D\rangle)=|D\rangle$; If $\hat{\omega}_{\delta}=\hat{D}_{\Delta}$, then $\hat{\omega}_{\delta}(|C\rangle)=|D\rangle$, $\hat{\omega}_{\delta}(|D\rangle)=|C\rangle . \hat{I}$ denotes "Not flip", $\hat{D}_{N}$ denotes "Flip".

3) The two sides of a card are denoted as Side 0 and Side 1 . The message written on the Side 0 (or Side 1 ) of card $j^{\delta}(0)$ is denoted as $\operatorname{card}\left(j^{\delta}(0), 0\right)$ (or $\left.\operatorname{card}\left(j^{\delta}(0), 1\right)\right)$. A typical card written by agent $j^{\delta}(0)$ is described as $c_{j^{\delta}(0)}=$ $\left(\operatorname{card}\left(j^{\delta}(0), 0\right), \operatorname{card}\left(j^{\delta}(0), 1\right)\right) \in \Theta \times A \times \mathbb{Z}_{+} \times \Theta \times A \times \mathbb{Z}_{+}$.

4) There is a device that can measure the state of $\Delta$ coins and send messages to the designer.

\subsection{Condition $\lambda^{S P E}$}

Given $\Delta \geq 2$ agents, consider the payoff to the $\Delta$-th agent, we denote by $\$_{C} \cdots C C$ the expected payoff when all $\Delta$ agents choose $\hat{I}$ (the corresponding collapsed state is $|C \cdots C C\rangle$ ), and denote by $\$_{C \cdots C D}$ the expected payoff when the $\Delta$ th agent chooses $\hat{D}_{\Delta}$ and the first $\Delta-1$ agents choose $\hat{I}$ (the corresponding collapsed state is $|C \cdots C D\rangle)$. $\$_{D \cdots D D}$ and $\$_{D \cdots D C}$ are defined similarly.

Definition 2: Given an SCC $f$ that satisfies Condition multi- $\alpha$, define Condition $\lambda^{S P E}$ as follows:

1) $\lambda_{1}^{S P E}$ : For the profiles $\theta, \phi$ specified in Condition multi- $\alpha$ and the outcome $a \in f(\theta)-f(\phi), a R^{j}(\phi) b(b \in f(\phi))$ for every $j \in J$, and $a P^{i}(\phi) b$ for at least 
one $i \in J$.

2) $\lambda_{2}^{S P E}$ : Consider the payoff to the $\Delta$-th agent, $\$_{C \cdots C C}>\$_{D \cdots D D}$, i.e., he $/$ she prefers the expected payoff of a certain outcome (generated by rule (1)) to the expected payoff of an uncertain outcome (generated by rule (2)).

3) $\lambda_{3}^{S P E}$ : Consider the payoff to the $\Delta$-th agent,

$\$_{C \cdots C C}>\$_{C \cdots C D}\left[1-\sin ^{2} \gamma \sin ^{2}(\pi / \Delta)\right]+\$_{D \cdots D C} \sin ^{2} \gamma \sin ^{2}(\pi / \Delta)$.

\subsection{Working steps of the quantum stage mechanism}

In the beginning of the traditional stage mechanism, each agent $j \in J$ directly announces a message $m^{j}$ (i.e., the triplet $\left(\theta^{j}, a^{j}, n^{j}\right)$ ) to the designer, then $N$ agents participate the stage mechanism as specified by rules (1)-(5). As a comparison, in the beginning of a quantum stage mechanism, the message $m^{j}$ $(j \in J)$ is generated by another way.

The setup of the quantum stage mechanism is depicted in Fig. 1. The working steps of a quantum stage mechanism are described as follows:

Step 1: Given an SCC $f$ and a profile $\xi \in \Theta$, if $f$ satisfies Condition multi- $\alpha$ and $\lambda^{S P E}$, and $\xi$ is equal to the profile $\phi$ specified in Condition $\lambda_{1}^{S P E}$, then go to Step 3.

Step 2: Each agent $j \in J$ sets $m^{j}=\left(\theta^{j}, a^{j}, n^{j}\right)$ (where $\theta^{j} \in \Theta, a^{j} \in A$, $\left.n^{j} \in \mathbb{Z}_{+}\right)$. Go to Step 9 .

Step 3: The state of each quantum $\operatorname{coin} \delta(1 \leq \delta \leq \Delta)$ is set as $|C\rangle$. The initial state of the $\Delta$ quantum coins is $\left|\psi_{0}\right\rangle=\underbrace{|C \cdots C C\rangle}_{\Delta}$.

Step 4: Each agent $j^{\delta}(0)(1 \leq \delta \leq \Delta)$ sets $c_{j \delta(0)}=((\theta, a, *),(\phi, *, *))$, where $\theta, \phi, a$ are specified in Condition $\lambda_{1}^{S P E}$. Each agent $j \in J-\bigcup_{\delta=1}^{\Delta} j^{\delta}(0)$ sets $c_{j}=\left((\theta, a, *),\left(\theta^{j}, a^{j}, n^{j}\right)\right)$.

Step 5: Let $\Delta$ quantum coins be entangled by $\hat{J} .\left|\psi_{1}\right\rangle=\hat{J}\left|\psi_{0}\right\rangle$.

Step 6: Each agent $j^{\delta}(0)$ independently performs a local unitary operation $\hat{\omega}_{\delta}$ on his/her own quantum coin. $\left|\psi_{2}\right\rangle=\left[\hat{\omega}_{1} \otimes \cdots \otimes \hat{\omega}_{\Delta}\right] \hat{J}\left|\psi_{0}\right\rangle$.

Step 7: Let $\Delta$ quantum coins be disentangled by $\hat{J}^{+} \cdot\left|\psi_{3}\right\rangle=\hat{J}^{+}\left[\hat{\omega}_{1} \otimes \cdots \otimes\right.$ $\left.\hat{\omega}_{\Delta}\right] \hat{J}\left|\psi_{0}\right\rangle$.

Step 8: The device measures the state of $\Delta$ quantum coins. For every $1 \leq$ $\delta \leq \Delta$, if the state of quantum coin $\delta$ is $|C\rangle$ (or $|D\rangle)$, then the device sends $\operatorname{card}\left(j^{\delta}(k), 0\right)$ (or $\left.\operatorname{card}\left(j^{\delta}(k), 1\right)\right)$ as $m^{j^{\delta}(k)}\left(0 \leq k \leq l^{\delta}\right)$ to the designer. For each agent $j \in J-\bigcup_{\delta=1}^{\Delta} \bigcup_{k=0}^{l^{\delta}} j^{\delta}(k)$, the device sends $m^{j}=\left(\theta, a, n^{j}\right)$ to the designer.

Step 9: The designer receives the overall message $m=\left(m^{1}, \cdots, m^{N}\right)$.

Step 10: The agents and the designer continue to participate the rules (1)-(5) of the traditional stage mechanism. END. 


\subsection{New result for subgame perfect implementation}

Proposition 1: For $N \geq 3$, consider an SCC $f$ that satisfies NVP and Condition $\alpha$, if $f$ satisfies multi- $\alpha$ and $\lambda^{S P E}$, then $f$ is not subgame perfect implementable by using the quantum stage mechanism.

Proof: Consider the profiles $\theta, \phi$ specified in $\lambda_{1}^{S P E}$. Since $f$ satisfies Condition multi- $\alpha$ and $\lambda^{S P E}$, then the quantum stage mechanism enters Step 3, and there exist $2 \leq \Delta \leq N$ sequences of agents that satisfy (i)-(iv) of Condition multi- $\alpha$. At first sight, each agent $j^{\delta}(0)(1 \leq \delta \leq \Delta)$ has incentives to unilaterally deviate from $(\theta, a, *)$ to $(\phi, *, *)$ in order to obtain his/her $\phi$-maximal outcome (as we have seen in Page 294, 3).

Consider the payoff to the $\Delta$-th agent (denoted as Laura), when she plays $\hat{\omega}(\xi, \eta)$ while the first $\Delta-1$ agents play $\hat{C}_{\Delta}=\hat{\omega}(0, \pi / \Delta)$, according to Refs. [5]11],

$$
\begin{aligned}
\left\langle \$_{\text {Laura }}\right\rangle & =\$_{C \cdots C C} \cos ^{2}(\xi / 2)\left[1-\sin ^{2} \gamma \sin ^{2}(\eta-\pi / \Delta)\right] \\
& +\$_{C \cdots C D} \sin ^{2}(\xi / 2)\left[1-\sin ^{2} \gamma \sin ^{2}(\pi / \Delta)\right] \\
& +\$_{D \cdots D C} \sin ^{2}(\xi / 2) \sin ^{2} \gamma \sin ^{2}(\pi / \Delta) \\
& +\$_{D \cdots D D} \cos ^{2}(\xi / 2) \sin ^{2} \gamma \sin ^{2}(\eta-\pi / \Delta)
\end{aligned}
$$

Since Condition $\lambda_{2}^{S P E}$ is satisfied, then $\$_{C \cdots C C}>\$_{D \cdots D D}$, Laura chooses $\eta=$ $\pi / \Delta$ to minimize $\sin ^{2}(\eta-\pi / \Delta)$. As a result,

$$
\begin{aligned}
\left\langle \$_{\text {Laura }}\right\rangle & =\$_{C \cdots C C} \cos ^{2}(\xi / 2) \\
& +\$_{C \cdots C D} \sin ^{2}(\xi / 2)\left[1-\sin ^{2} \gamma \sin ^{2}(\pi / \Delta)\right] \\
& +\$_{D \cdots D C} \sin ^{2}(\xi / 2) \sin ^{2} \gamma \sin ^{2}(\pi / \Delta)
\end{aligned}
$$

Since Condition $\lambda_{3}^{S P E}$ is satisfied, then Laura prefers $\xi=0$, which leads to $\left\langle \$_{\text {Laura }}\right\rangle=\$_{C \ldots C C}$. In this case, $\hat{\omega}_{\text {Laura }}(\xi, \eta)=\hat{\omega}(0, \pi / \Delta)=\hat{C}_{\Delta}$.

By symmetry, in Step 6, each agent $j^{\delta}(0)(1 \leq \delta \leq \Delta)$ chooses $\hat{\omega}_{\delta}=\hat{C}_{\Delta}$. In Step 8, the collapsed state of each quantum coin $\delta$ is $|C \cdots C C\rangle$, and $m^{j}=$ $(\theta, a, *)$ for each agent $j \in J$. No agent $j^{\delta}(0)(1 \leq \delta \leq \Delta)$ is willing to deviate from $(\theta, a, *)$ to $(\phi, *, *)$ in the quantum stage mechanism. Consequently, in profile $\phi, a \in f(\theta)-f(\phi)$ can be implemented in subgame perfect equilibrium by rule (1). Therefore, $f$ is not subgame perfect implementable.

Let us reconsider Example 1. $f$ satisfies Condition multi- $\alpha$ and $\lambda_{1}^{S P E} \cdot \theta=$ $\theta_{1}, \phi=\theta_{2}$. The quantum stage mechanism will enter Step 3 when the true profile is $\theta_{2}$. Each agent $j \in\{$ Alice, Lily $\}$ sets $c_{j}=\left(\left(\theta_{1}, u_{1}, *\right),\left(\theta_{2}, *, *\right)\right)$. Each agent $j \in\{$ Apple,Cindy $\}$ sets $c_{j}=\left(\left(\theta_{1}, u_{1}, *\right),\left(\theta^{j}, a^{j}, n^{j}\right)\right)$. For any agent $j \in\{$ Alice, Lily $\}$, let her be the last agent. Consider the payoff to the fourth agent, suppose $\$_{C C C C}=3$ (the corresponding outcome is $u_{1}$ ), $\$_{C C C D}=5$ (the corresponding outcome is $u_{4}$ if $j=$ Alice, and $u_{3}$ if $j=$ Lily), $\$_{D D D C}=0$ (the corresponding outcome is $u_{3}$ if $j=$ Alice, and $u_{4}$ if $j=$ Lily), $\$_{D D D D}=1$ 
(the corresponding outcome is uncertain among $A$ ). Hence, Condition $\lambda_{2}^{S P E}$ is satisfied, and Condition $\lambda_{3}^{S P E}$ becomes: $3 \geq 5\left[1-\sin ^{2} \gamma \sin ^{2}(\pi / 2)\right]$. If $\sin ^{2} \gamma \geq$ 0.4 , Condition $\lambda_{3}^{S P E}$ is satisfied. According to Proposition 1, in profile $\theta_{2}$, the Pareto-efficient outcome $u_{1}$ is generated in subgame perfect equilibrium by using the quantum stage mechanism.

\section{An algorithmic stage mechanism}

Following Ref. [7], in this section we will propose an algorithmic stage mechanism to help agents benefit from the quantum stage mechanism immediately. In the beginning, we cite the matrix representations of quantum states from Ref. [7].

\subsection{Matrix representations of quantum states}

In quantum mechanics, a quantum state can be described as a vector. For a two-level system, there are two basis vectors: $(1,0)^{T}$ and $(0,1)^{T}$. In the beginning, we define:

$$
\begin{aligned}
& |C\rangle=\left[\begin{array}{l}
1 \\
0
\end{array}\right], \quad \hat{I}=\left[\begin{array}{ll}
1 & 0 \\
0 & 1
\end{array}\right], \quad \hat{\sigma}_{x}=\left[\begin{array}{ll}
0 & 1 \\
1 & 0
\end{array}\right],\left|\psi_{0}\right\rangle=\underbrace{|C \cdots C C\rangle}_{\Delta}=\left[\begin{array}{c}
1 \\
0 \\
\cdots \\
0
\end{array}\right]_{2^{\Delta} \times 1} \\
& \hat{J}=\cos (\gamma / 2) \hat{I}^{\otimes \Delta}+i \sin (\gamma / 2) \hat{\sigma}_{x}^{\otimes \Delta}
\end{aligned}
$$

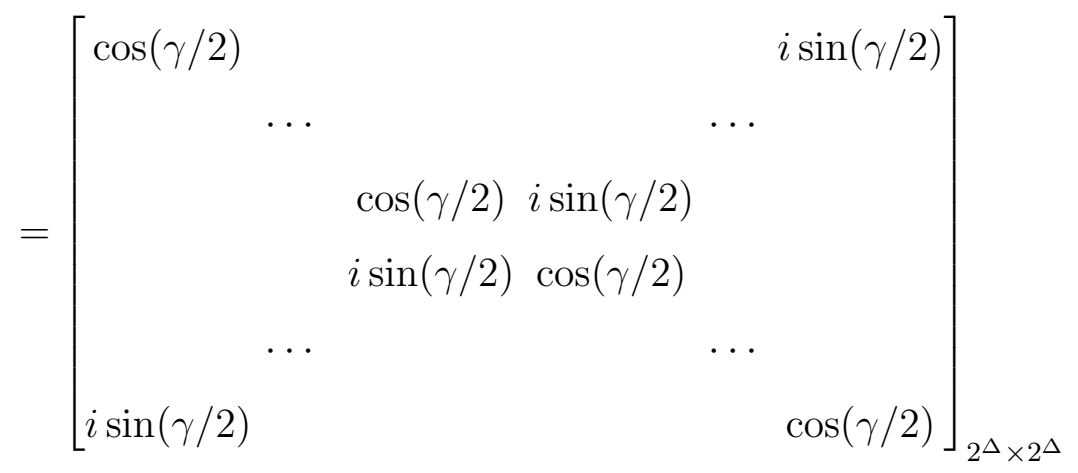




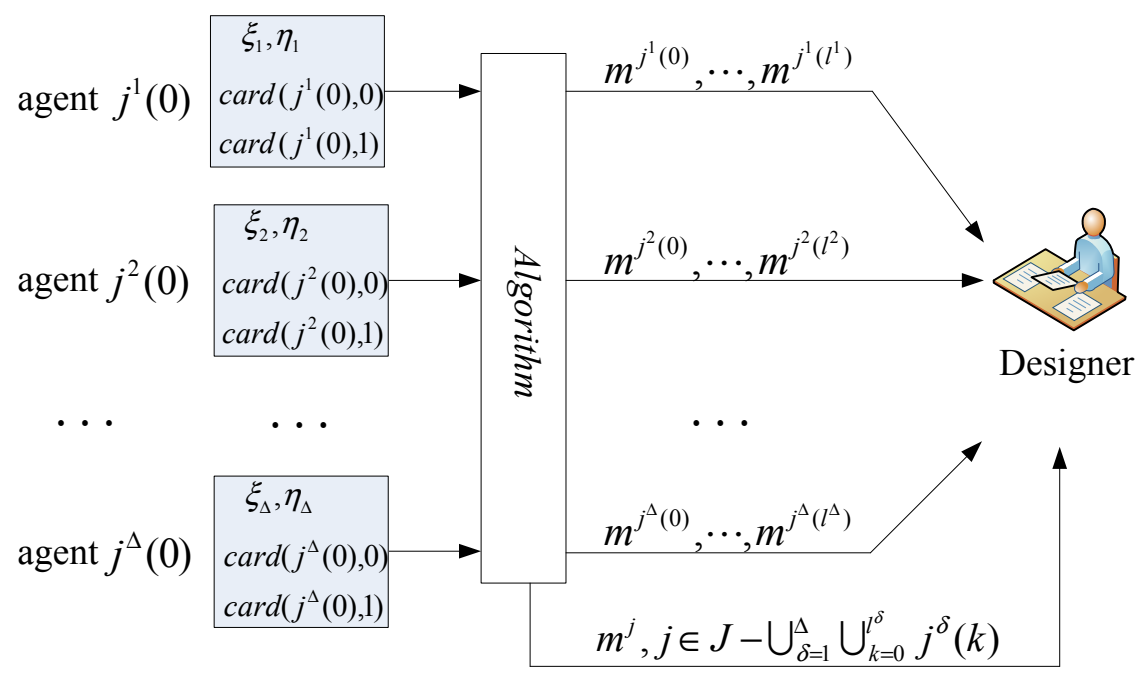

Fig. 2. The inputs and outputs of the algorithm.

For $\gamma=\pi / 2$,

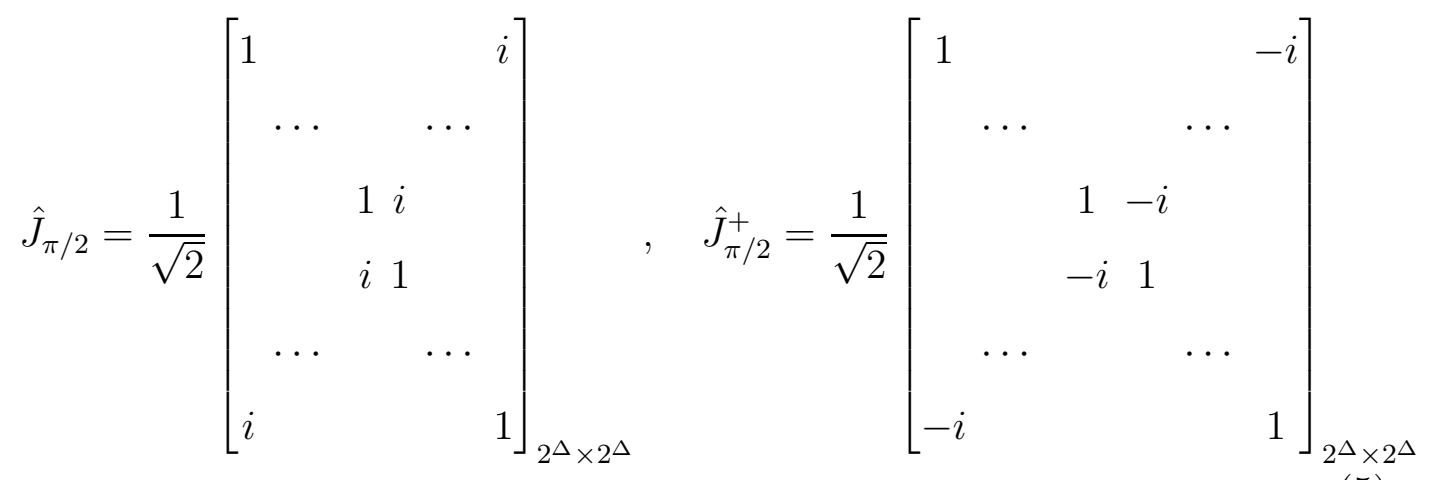

\subsection{An algorithm that simulates the quantum operations and measurements}

Similar to Ref. [7], in the following we will propose an algorithm that simulates the quantum operations and measurements in the quantum stage mechanism. The amendment here is that now the inputs and outputs are adjusted to the case of subgame perfect implementation. The factor $\gamma$ is also set as its maximum $\pi / 2$. For $\Delta$ agents, the inputs and outputs of the algorithm are illustrated in Fig. 2. The Matlab program is given in Fig. 3.

\section{Inputs:}

1) $\xi_{\delta}, \eta_{\delta}, \delta=1, \cdots, \Delta$ : the parameters of agent $j^{\delta}(0)$ 's local operation $\hat{\omega}_{\delta}$, $\xi_{\delta} \in[0, \pi], \eta_{\delta} \in[0, \pi / 2]$.

$2) \operatorname{card}\left(j^{\delta}(0), 0\right), \operatorname{card}\left(j^{\delta}(0), 1\right), \delta=1, \cdots, \Delta$ : the information written on the two sides of agent $j^{\delta}(0)$ 's card, where $\operatorname{card}\left(j^{\delta}(0), 0\right), \operatorname{card}\left(j^{\delta}(0), 1\right) \in \Theta \times A \times$ $\mathbb{Z}_{+}$. 


\section{Outputs:}

$m^{j^{\delta}(k)}\left(\delta=1, \cdots, \Delta, k=0, \cdots, l^{\delta}\right)$ : the agent $j^{\delta}(k)$ 's message that is sent to the designer, $m^{j^{\delta}(k)} \in \Theta \times A \times \mathbb{Z}_{+}$.

\section{Procedures of the algorithm:}

Step 1: Reading parameters $\xi_{\delta}$ and $\eta_{\delta}$ from each agent $j^{\delta}(0)(\delta=1, \cdots, \Delta)$ (See Fig. 3(a)).

Step 2: Computing the leftmost and rightmost columns of $\hat{\omega}_{1} \otimes \hat{\omega}_{2} \otimes \cdots \otimes \hat{\omega}_{\Delta}$ (See Fig. 3(b)).

Step 3: Computing the vector representation of $\left|\psi_{2}\right\rangle=\left[\hat{\omega}_{1} \otimes \cdots \otimes \hat{\omega}_{\Delta}\right] \hat{J}_{\pi / 2}\left|\psi_{0}\right\rangle$. Step 4: Computing the vector representation of $\left|\psi_{3}\right\rangle=\hat{J}_{\pi / 2}^{+}\left|\psi_{2}\right\rangle$.

Step 5: Computing the probability distribution $\left\langle\psi_{3} \mid \psi_{3}\right\rangle$ (See Fig. 3(c)).

Step 6: Randomly choosing a "collapsed" state from the set of all $2^{\Delta}$ possible states $\{|C \cdots C C\rangle, \cdots,|D \cdots D D\rangle\}$ according to the probability distribution $\left\langle\psi_{3} \mid \psi_{3}\right\rangle$.

Step 7: For each agent $j^{\delta}(k)\left(\delta=1, \cdots, \Delta, k=0, \cdots, l^{\delta}\right)$, the algorithm sends $\operatorname{card}\left(j^{\delta}(k), 0\right)$ (or $\left.\operatorname{card}\left(j^{\delta}(k), 1\right)\right)$ as the message $m^{j^{\delta}(k)}$ to the designer if the $\delta$-th basis vector of the "collapsed" state is $|C\rangle$ (or $|D\rangle)$.

Step 8: For each agent $j \in J-\bigcup_{\delta=1}^{\Delta} \bigcup_{k=0}^{l^{\delta}} j^{\delta}(k)$, the algorithm sends $m^{j}=$ $\operatorname{card}(j, 0)$ to the designer (See Fig. $3(\mathrm{~d}))$.

\subsection{An algorithmic version of the quantum stage mechanism}

Since the entanglement measure $\gamma$ is set as its maximum $\pi / 2$, the Condition $\lambda^{S P E}$ shall be revised as $\lambda^{\prime S P E}$.

Definition 6: Given an SCC $f$ that satisfies Condition multi- $\alpha$, define Condition $\lambda^{\prime S P E}$ as follows:

1) $\lambda_{1}^{\prime S P E}$ and $\lambda_{2}^{\prime S P E}$ are the same as $\lambda_{1}^{S P E}$ and $\lambda_{2}^{S P E}$ respectively.

2) $\lambda_{3}^{\prime S P E}$ : Consider the payoff to the $\Delta$-th agent, $\left.\$_{C \cdots C C}>\$_{C \cdots C D} \cos ^{2}(\pi / \Delta)\right]+$ $\$_{D \cdots D C} \sin ^{2}(\pi / \Delta)$.

Following Ref. [7], after quantum operations and measurements in quantum stage mechanism are replaced by an algorithm, the quantum stage mechanism shall be revised as an algorithmic stage mechanism. The working steps of an algorithmic stage mechanism are described as follows:

Step 1: Given an SCC $f$ and a profile $\xi \in \Theta$, if $f$ satisfies Condition multi- $\alpha$ and $\lambda^{\prime S P E}$, and $\xi$ is equal to the profile $\phi$ specified in Condition $\lambda_{1}^{\prime S P E}$, then go to Step 3.

Step 2: Each agent $j \in J$ sets $m^{j}=\left(\theta^{j}, a^{j}, n^{j}\right)$ (where $\theta^{j} \in \Theta, a^{j} \in A$, $\left.n^{j} \in \mathbb{Z}_{+}\right)$. Go to Step 6 .

Step 3: Each agent $j^{\delta}(0)(\delta=1, \cdots, \Delta)$ sets $c_{j^{\delta}(0)}=((\theta, a, *),(\phi, *, *))$, 
where $\theta, \phi, a$ are specified in $\lambda_{1}^{\prime S P E}$; Each agent $j \in J-\bigcup_{\delta=1}^{\Delta} j^{\delta}(0)$ sets $c_{j}=$ $\left((\theta, a, *),\left(\theta^{j}, a^{j}, n^{j}\right)\right)$.

Step 4: Each agent $j^{\delta}(0)(\delta=1, \cdots, \Delta)$ submits $\xi_{\delta}, \eta_{\delta}, \operatorname{card}\left(j^{\delta}(0), 0\right)$ and $\operatorname{card}\left(j^{\delta}(0), 1\right)$ to the algorithm.

Step 5: The algorithm runs in a computer and outputs messages $m^{j}(j \in J)$ to the designer.

Step 6: The designer receives the overall message $m=\left(m^{1}, \cdots, m^{N}\right)$.

Step 7: The agents and the designer continue to participate the rules (1)-(5) of the traditional stage mechanism. END.

Proposition 2: For $N \geq 3$, consider an SCC $f$ that satisfies NVP and Condition $\alpha$, if $f$ satisfies Condition multi- $\alpha$ and $\lambda^{\prime S P E}$, then $f$ is not subgame perfect implementable by using the algorithmic stage mechanism.

The proof of proposition 2 is straightforward according to Proposition 1 and Ref. [7]. Note: Although the algorithmic stage mechanism stems from quantum mechanics, it is completely classical that can be run in a computer. In addition, Condition $\lambda^{\prime S P E}$ is also a classical condition.

\section{Conclusions}

This paper follows the series of papers on quantum mechanism [5,7,8,9]. In this paper, the quantum and algorithmic mechanisms in Refs. [5,7] are generalized to subgame perfect implementation. In Example 1, we show a Pareto-inefficient SCC $f$ that satisfies NVP and Condition $\alpha$, but does not satisfies monotonicity. According to the traditional stage mechanism [3], $f$ can be subgame perfect implemented. However, by virtue of a quantum stage mechanism, the Paretoinefficient SCC $f$ can not be implemented in subgame perfect equilibrium. Although current experimental technologies restrict the quantum stage mechanism to be commercially available, for small-scale cases (e.g., $\Delta \leq 20$ [7]), the algorithmic stage mechanism can help agents benefit from quantum stage mechanism immediately.

\section{Acknowledgments}

The author is very grateful to Ms. Fang Chen (Alice), Hanyue Wu (Apple), Hanxing $\mathrm{Wu}$ (Lily) and Hanchen Wu (Cindy) for their great support. 


\section{References}

[1] E. Maskin, Nash equilibrium and welfare optimality, Rev. Econom. Stud. 66 (1999) 23-38.

[2] J. Moore and R. Repullo, Subgame perfect implementation. Econometrica, 56 (1988) 1191-1220.

[3] D. Abreu and A. Sen, Subgame perfect implementation: a necessary and almost sufficient condition. Journal of Economic Theory, 50 (1990) 285-299.

[4] H. Vartiainen, Subgame perfect implementation: a full characterization. Journal of Economic Theory, 133 (2007) 111-126.

[5] H. Wu, Quantum mechanism helps agents combat "bad" social choice rules. International Journal of Quantum Information, 2010 (accepted). http://arxiv.org/abs/1002.4294

[6] T.D. Ladd, F. Jelezko, R. Laflamme, Y. Nakamura, C. Monroe and J.L. O'Brien, Quantum computers, Nature, 464 (2010) 45-53.

[7] H. Wu, On amending the sufficient conditions for Nash implementation. Theoretical Computer Science, 2011 (submitted). http://arxiv.org/abs/1004.5327

[8] H. Wu, Two-agent Nash implementation: A new result. Games and Economic Behaviour, 2011 (submitted). http://arxiv.org/abs/1005.2135

[9] H. Wu, Quantum and algorithmic Bayesian mechanisms. http://arxiv.org/abs/1104.0471

[10] R. Serrano, The theory of implementation of social choice rules, SIAM Review 46 (2004) 377-414.

[11] A.P. Flitney and L.C.L. Hollenberg, Nash equilibria in quantum games with generalized two-parameter strategies, Phys. Lett. A 363 (2007) 381-388. 


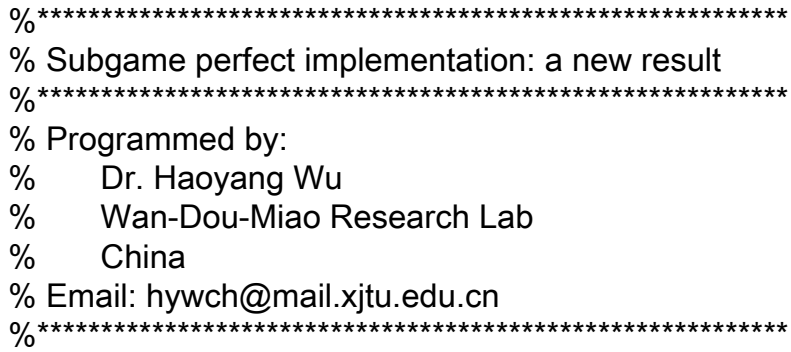

start_time = cputime;

$\% \mathrm{~N}$ : the number of agents. See the Example 1 given in Section 3.

$\%$ Let $\mathrm{J}=\{1,2,3,4\}$ represent \{Alice, Apple, Lily, Cindy\}

$\mathrm{N}=4$;

agent_name $=$ cell $(\mathrm{N}, 1)$;

agent_name $\{1\}=$ 'Alice';

agent_name $\{2\}=$ 'Apple';

agent_name $\{3\}=$ 'Lily';

agent_name $\{4\}=$ 'Cindy';

$\% \Delta:$ the number of sequences of agents specified in Condition multi- $\alpha$

Delta $=2$;

$\% l^{\delta}$ : the length of the $\delta$-th sequence

Idelta $=$ zeros $(2,1)$;

Idelta(1) $=1 ; \quad \% l^{1}=1$

$\operatorname{ldelta}(2)=1 ; \quad \% l^{2}=1$

$\% j^{\delta}$ : the sequences of agents $j^{\delta}(0), \cdots, j^{\delta}\left(l^{\delta}\right)$

jdelta $=$ zeros $(2,2)$;

jdelta $(1,1)=1 ; \quad \% j^{1}(0)=$ Alice

jdelta $(1,2)=2 ; \quad \% j^{1}(1)=$ Apple

jdelta $(2,1)=3 ; \quad \% j^{2}(0)=$ Lily

jdelta $(2,2)=4 ; \quad \% j^{2}(1)=$ Cindy

$\% \gamma:$ the coefficient of entanglement. We simply set gamma to its maximum $\pi / 2$

gamma $=\mathrm{pi} / 2$;

$\%(\xi, \eta)$ denote the array of parameters of each agent $j^{\delta}(0)$ 's unitary operation, $\delta=1, \cdots, \Delta$

$\mathrm{xi}=$ zeros $($ Delta, 1$)$;

eta $=$ zeros $($ Delta, 1$)$;

$\%$ Reading parameters $\xi_{\delta}$ and $\eta_{\delta}$ from each agent $j^{\delta}(0), \delta=1, \cdots, \Delta$

$\mathrm{xi}(1)=0$;

eta(1) $=\mathrm{pi} / 2 ; \quad \% \hat{\omega}_{1}=\hat{C}_{\Delta}=\hat{\omega}(0, \pi / 2)$

$\mathrm{xi}(2)=0$;

eta $(2)=\mathrm{pi} / 2 ; \quad \% \hat{\omega}_{2}=\hat{C}_{\Delta}=\hat{\omega}(0, \pi / 2)$

Fig. 3 (a). Reading each agent $j^{\delta}(0)$ 's parameters $\xi_{\delta}$ and $\eta_{\delta}, \delta=1, \cdots, \Delta$. 


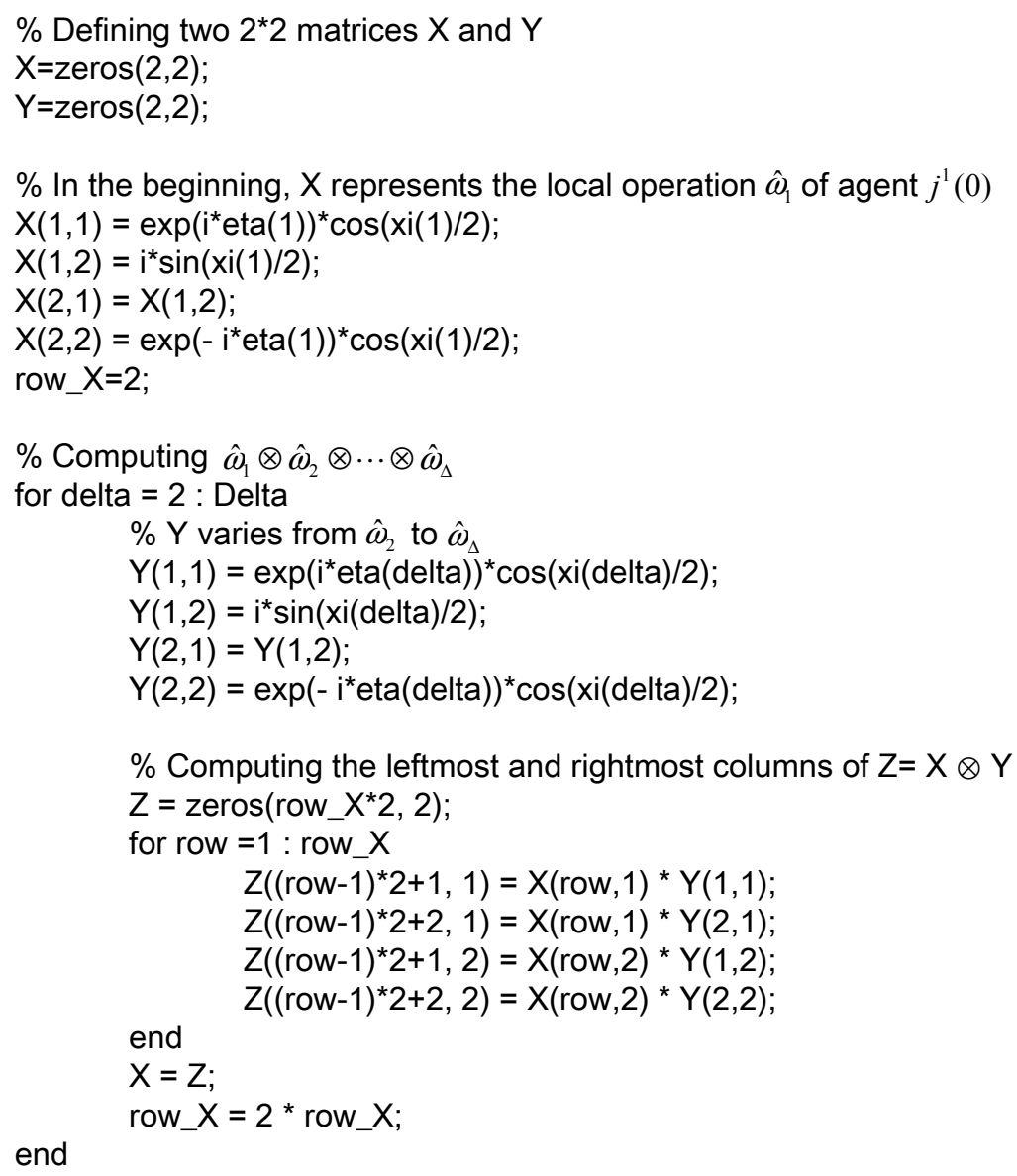

$\%$ Now the matrix $X$ contains the leftmost and rightmost columns of $\hat{\omega}_{1} \otimes \hat{\omega}_{2} \otimes \cdots \otimes \hat{\omega}_{\Delta}$

Fig. 3 (b). Computing the leftmost and rightmost columns of $\hat{\omega}_{1} \otimes \hat{\omega}_{2} \otimes \cdots \otimes \hat{\omega}_{\Delta}$

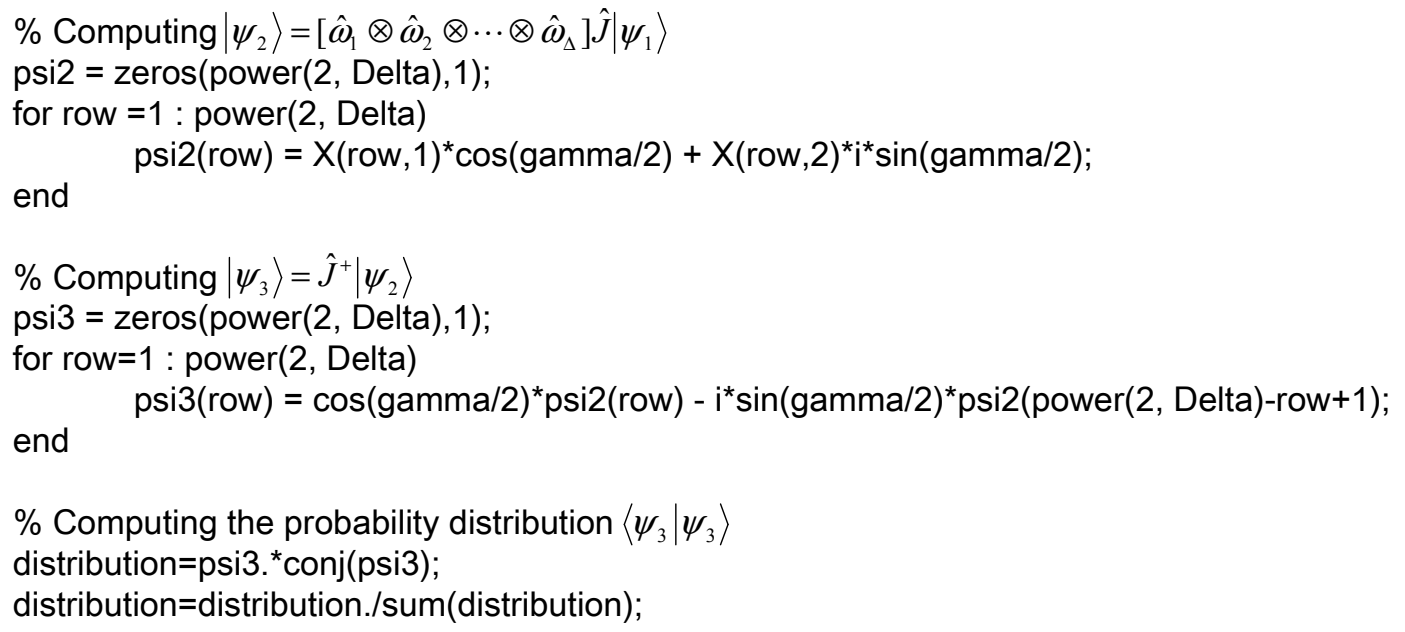

Fig. 3 (c). Computing $\left|\psi_{2}\right\rangle,\left|\psi_{3}\right\rangle,\left\langle\psi_{3} \mid \psi_{3}\right\rangle$. 


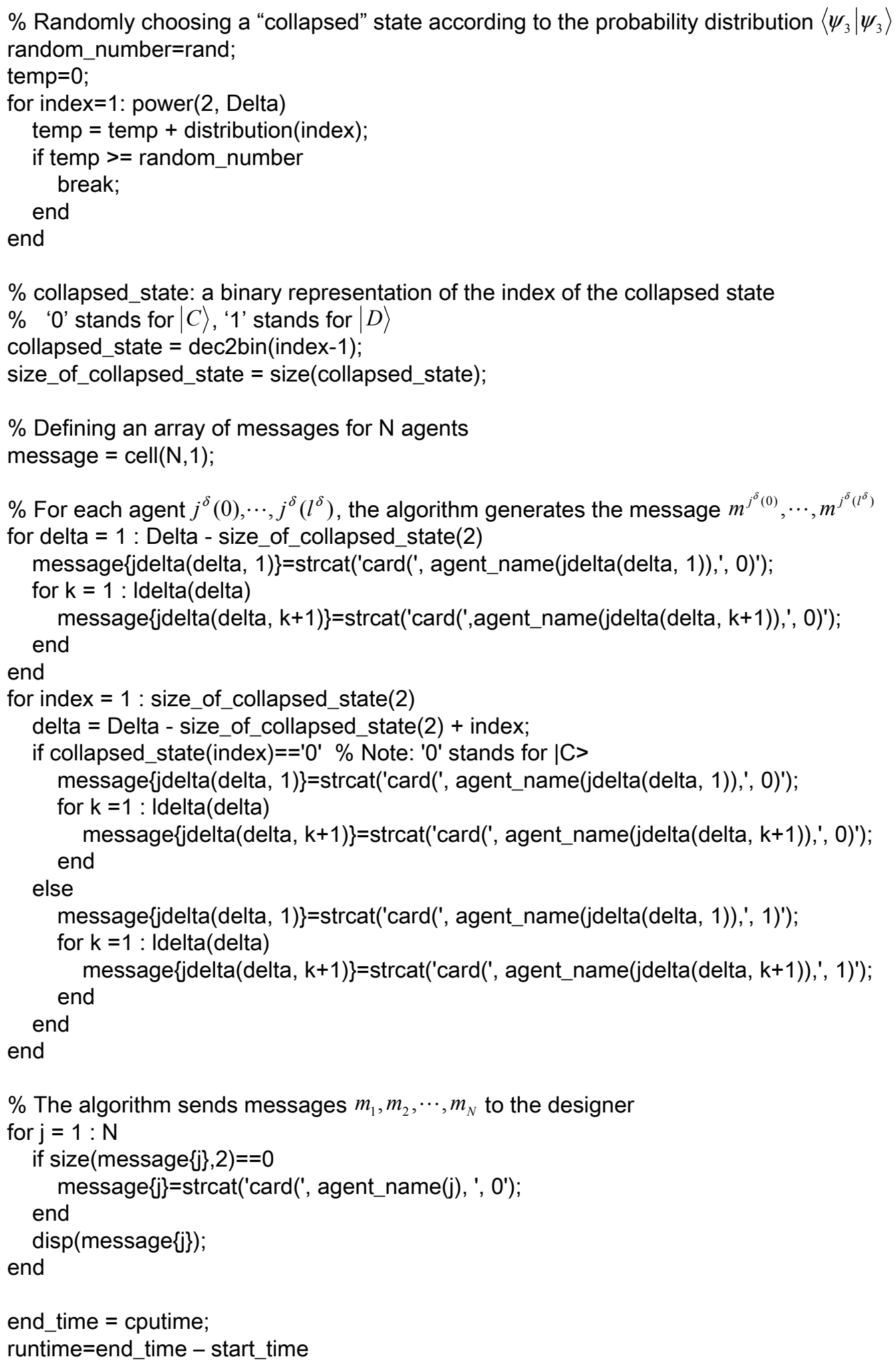

Fig. 3 (d). Computing all messages $m_{1}, m_{2}, \cdots, m_{N}$. 